\title{
HEPATIC SAFETY AND POSSIBLE HEPATOTOXIC ACTION OF THE METABOLITES OF SOME PHARMACEUTICAL DRUGS
}

\author{
Svetlana Georgieva \\ Department of Pharmaceutical Sciences, Faculty of Pharmacy, \\ Medical University of Varna
}

\begin{abstract}
Liver is the principle organ for maintaining the body's internal environment. There is currently no way to reimburse for the absence of liver function. Its major influence is on the flow of nutrients and controls the metabolism of carbohydrate, protein and fats. Drugs are an important cause of liver injury. More than 900 drugs, toxins, and herbs have been reported to cause liver injury. Certain medicinal agents, when taken in overdoses and sometimes even when introduced within therapeutic ranges, may injure the organ. Various types of drug induced liver diseases are acute-dose dependent liver damage, acute fatty infiltration, cholestatic jaundice, liver granulomas, active chronic hepatitis, liver cirrhosis, liver tumors etc. The ability to accurately predict the toxicity of drug candidates and their metabolites from their chemical structure is critical for guiding experimental drug discovery toward safer medicines. Considering the importance of druginduced hepatotoxicity as a major cause of liver damage, this article throws light on hepatic safety and possible hepatotoxic action of the metabolites of some pharmaceutical drugs by using in silico modeling.
\end{abstract}

Key words: drug-related hepatotoxicity, liver injury, pharmaceutical drugs, metabolites, QSAR Toolbox

\section{INTRODUCTION}

Hepatotoxicity is defined as the damage to the liver caused by drugs or chemicals. There are many substances capable of damaging the liver with several very different mechanisms and, as such, damage cannot be considered an isolated pathology rather than a compounding of factors involved in its development (8).

Clinical presentation of hepatic toxicity varies considerably and encompasses asymptomatic or re-

Address for correspondence:

Svetlana Georgieva, PhD

Department of Pharmaceutical Sciences,

Faculty of Pharmacy, Medical University of Varna,

55 Marin Drinov Str., 9002 Varna, Bulgaria

e-mail:fotkova@abv.bg

Received: February 04, 2013

Accepted: March 22, 2013 versible alterations up to fulminant hepatic failure $(2,6)$. In addition, a toxic substance may sometimes present with different patterns of toxicity and, as such, it is not always possible to link a specific clinical pattern with a particular drug (10). Incrimination of a drug in liver symptoms requires a high degree of suspicion on the part of the physician. It includes a temporal relationship, awareness of the drug's hepatotoxic potential, differential diagnosis, exclusion of alternative causes of liver damage and ability to assess subtle data that favour a toxic etiology. Besides there are no specific analytical or clinical criteria for hepatotoxicity; exclusion of other possible etiologies being the only means of diagnosis currently available $(9,12)$.

Hepatotoxicity based on laboratory criteria may be classified as:

* acute hepato-cellular type: initially elevated ALT followed by increased levels of bilirubin 
and alkaline phosphatase (AlkPhos); the latter, however, being quite modest. The ALT/AlkPhos ratio (R) expressed as a function of the ULN values would be $R \geq 5$ (1). It describes patients with a presentation similar to that of acute viral hepatitis, together with general discomfort, asthenia, anorexia and nausea. Prognosis would vary substantially and is related to the extent of liver damage (16).

* acute cholestatic injury: initially increased AlkPhos values which are more prominent and precocious than those of ALT or AST and with a $R$ value $\leq 2$ (1).

This condition can be classified into two types:

* pure, soft or canalicular: clinical features include jaundice and pruritus. The condition is characterized by an increased conjugated bilirubin, AlkPhos and $\gamma$-glutamyl transpeptidase $(\gamma$-GT) with little or no impairment of serum transaminase values. Liver histology shows hepatocyte cholestasis and dilated biliary canaliculi with bile-plugs (7). The prognosis is good following laboratory and clinical normalization.

* hepatocanalicular: usually, it presents with abdominal pain, fever and shivering; hypersensitivity episodes being frequent (1). The condition is characterized by canalicular damage with hepatic inflammation and elevated serum transaminases. Prognosis is better than that of hepato-cellular lesions.

* mixed hepatic injury: it is used to describe the clinical and biological patterns which are intermediate between the hepato-cellular and cholestatic types, with features of either type predominating. Laboratory measurements show an elevated ALT $>2 x U L N$ with an increment of AlkPhos and $\mathrm{R}>2$ but $\mathrm{R}<5$ (1). Hypersensitivity features and a granulomatous reaction in the liver biopsy specimen are often observed (11).

Some drugs are not intrinsically toxic to the liver but can cause injury secondary to the production of a hepatotoxic drug metabolite, a process known as bioactivation $(3,5)$. Because gastrointestinal absorption is enhanced by lipid solubility, most xenobiotics are highly lipophilic compounds, which are poorly excreted by the kidneys (3). The liver plays a critical role in promoting the excretion of these compounds by transforming them into metabolites of greater water solubility.

Metabolic reactions are of two types, phase I and phase II $(3,5)$.

Phase I (oxidation, reduction, or hydrolysis) reactions typically occur first and enhance water solubility by generating hydroxyl, carboxy, or epoxide functional groups on the parent compound. These functional groups in turn facilitate phase II reactions, conjugation with glucuronate, sulfate, acetate, or glutathione moieties. Conjugation reactions generally serve to further enhance water solubility and renal excretion (3). Phase II reactions also play a role in the prevention of xenobiotic-induced liver injury as most conjugates are biologically inactive $(3,5,18)$. Disruption of normal phase II processes can lead to accumulation of hepatotoxic phase I metabolites.

The aim of this work is to predict the possible metabolites of some pharmaceutical drugs by a specialized software which can cause hepatotoxic action. This could help medical professionals to easily predict liver injury in patients with liver disease.

\section{MATERIAL AND METHODS Compounds}

In the present study, three pharmaceutical drugs (4) are investigated. They are presented in Table 1.

\section{OECD (Q)SAR Application Toolbox}

(Quantitative) Structure-Activity Relationships [(Q)SARs] are methods for estimating the properties of a chemical from its molecular structure. They have the potential to provide information about the hazards of chemicals while reducing time, monetary costs and animal testing currently needed. In order to facilitate practical application of (Q)SAR approaches in regulatory contexts by governments and industry and to improve their regulatory acceptance, the OECD (Q)SAR project has developed various outcomes such as the principles for the validation of (Q)SAR models, guidance documents and the QSAR Toolbox (15).

Metabolic pathways documented for 200 organic chemicals in different mammals are stored in a database format that allows easy computer-aided ac- 
Table 1. CAS number, name and structure of some antibiotics

\begin{tabular}{|l|l|l|l|l|}
\hline No & CAS number & Name of compound & Amoxicillin & \\
\hline 1. & $26787-78-0$ & Structure of compound \\
\hline 2. & $61-72-3$ & Cloxacillin & Penicillin & \\
\hline 3. & $1406-05-9$ & &
\end{tabular}

cess to the metabolism information. The collection includes chemicals of different classes, a variety of functionalities such aliphatic hydrocarbons, alicyclic rings, furans, halogenated hydrocarbons, aromatic hydrocarbons and haloaromatics, amines, nitroderivatives, and multifunctional compounds. In vivo and in vitro (predominantly, with liver microsomes as experimental systems) studies are used to analyze the metabolic fate of chemicals. Different sources, including monographs, scientific articles and public websites were used to compile the database $(13,15)$.

\section{RESULTS AND DISCUSSION}

Pharmaceutical drugs used by general practitioners frequently appear in adverse-event reports of drug-induced hepatotoxicity. Most cases are idiosyncratic (the adverse reaction cannot be predicted from the drug's pharmacological profile or from pre-clinical toxicology tests) and occur via an immunological reaction or in response to the presence of hepatotoxic metabolites.

Electrophilic intermediates/metabolites and parent chemicals can react with many different nucleophilic sites in cells. However, the electrophilic metabolites may not only react with nucleophilic sites in DNA but may also bind to proteins, RNA, and endogenous substances of lower molecular weight such as glutathione (14). The complexity of their reaction with various nucleophilic sites within cells and the reasons why different electrophilic reagents react at different sites are interpreted on the basis of the concepts of hard and soft electrophiles/ nucleophiles (hard and soft acids/bases) (17).

Some examples of compounds belonging to different drug classes are elucidated for their probable metabolic activation in liver (observed and predicted) and protein and DNA binding, respectively (Table 2).

\section{CONCLUSION}

When an antibiotic reaches the clinical development phase, the attributability of any rare but servere side effects must be thoroughly investigated. When a problem is discovered shortly before or after marketing, specific clinical studies are required to determine the degree of risk. The process can be assisted by strategies and decision trees drawnup by expert groups. This can lead to a change in the summary of product characteristics, or, after a re-assessment of the risk-benefit ratio, to market withdrawal by public health authorities.

\section{REFERENCES}

1. Bénichou, C. Criteria for drug-induced liver disorders. Report of an International Consensus Meeting.- J. Hepatol., 11, 1990, No 2, 272-276. 
Table 2. Probable metabolic activation of some antibiotics by (Q)SAR Application Toolbox

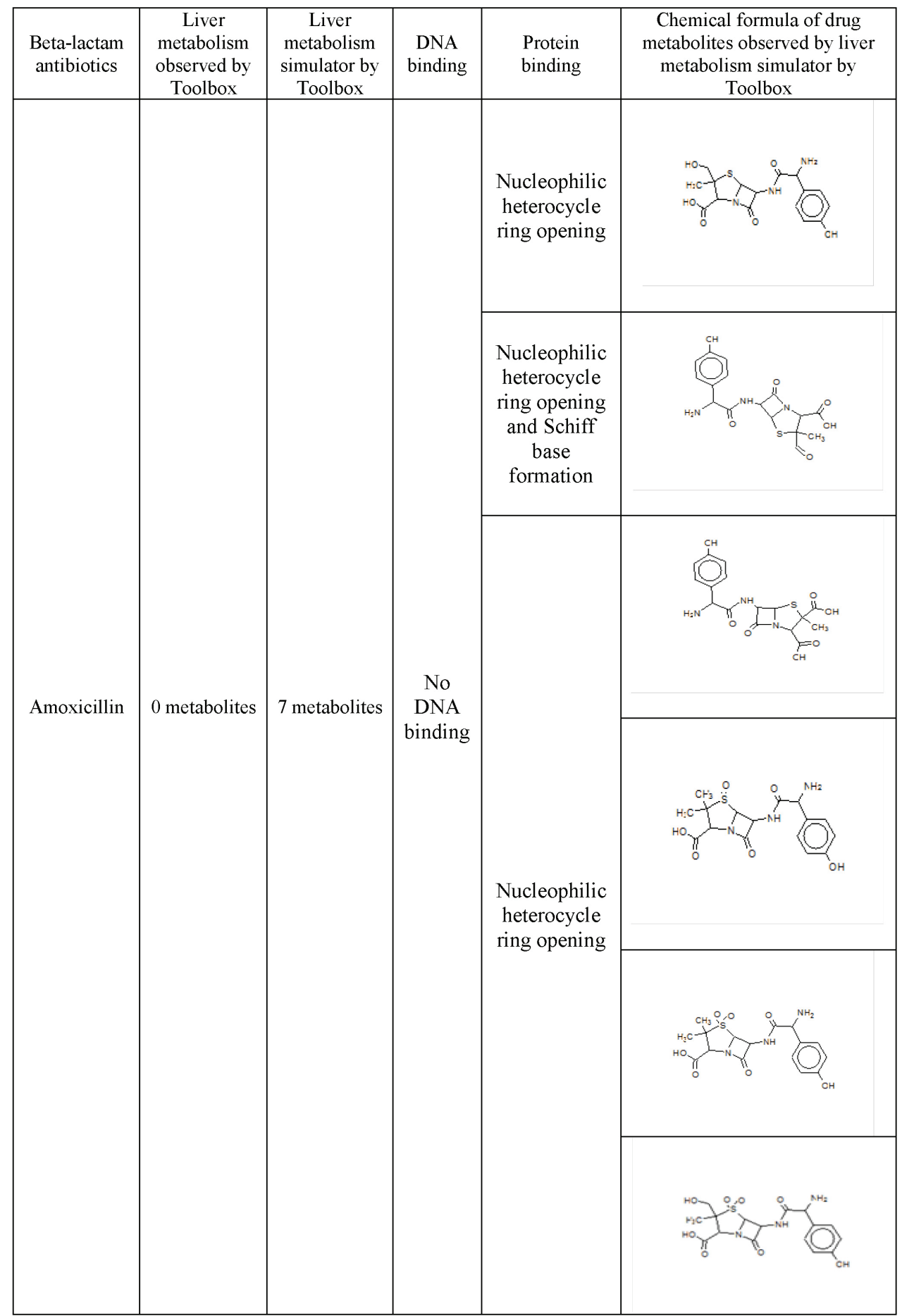


Hepatic safety and possible hepatotoxic action of the metabolites of some pharmaceutical drugs

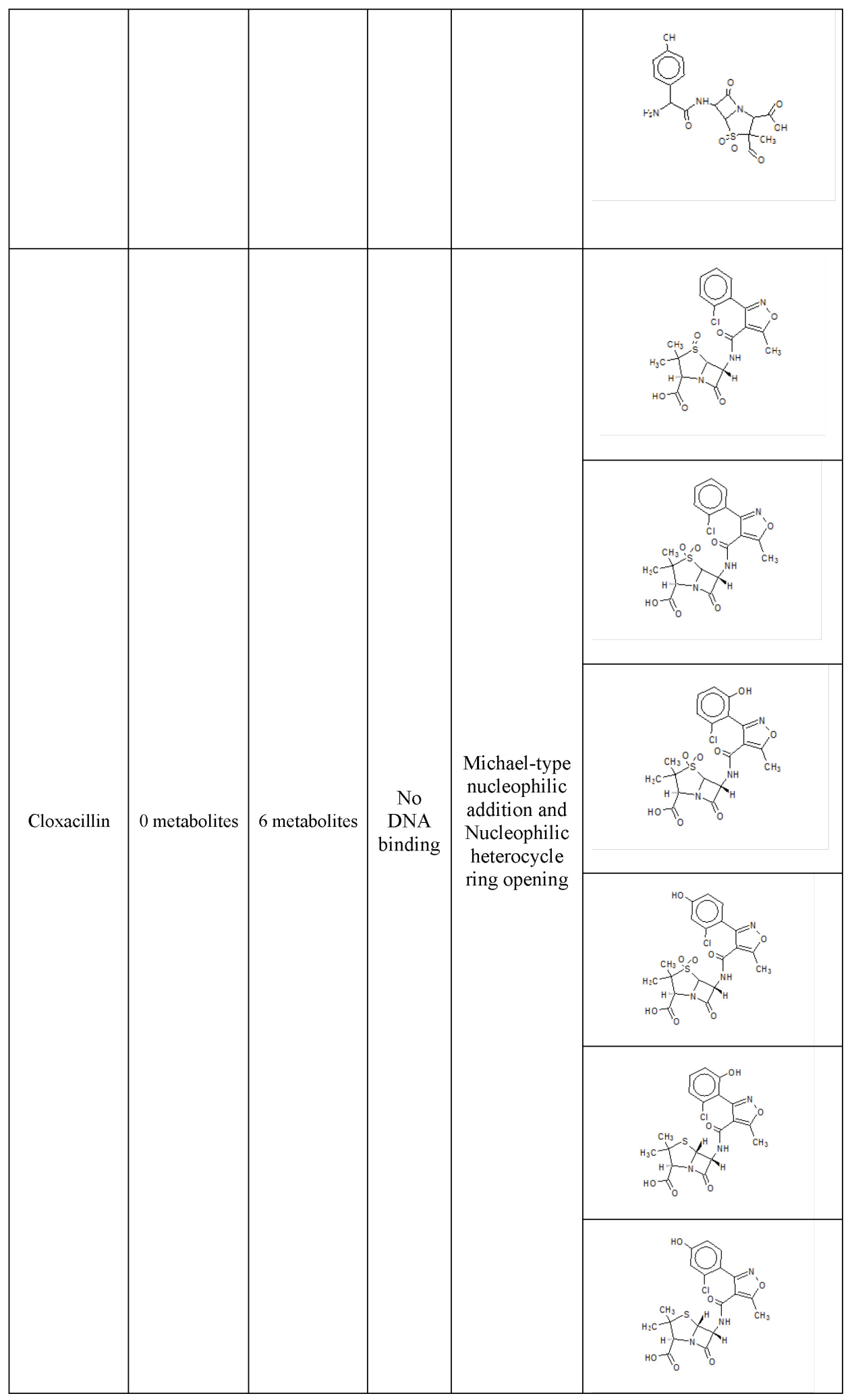




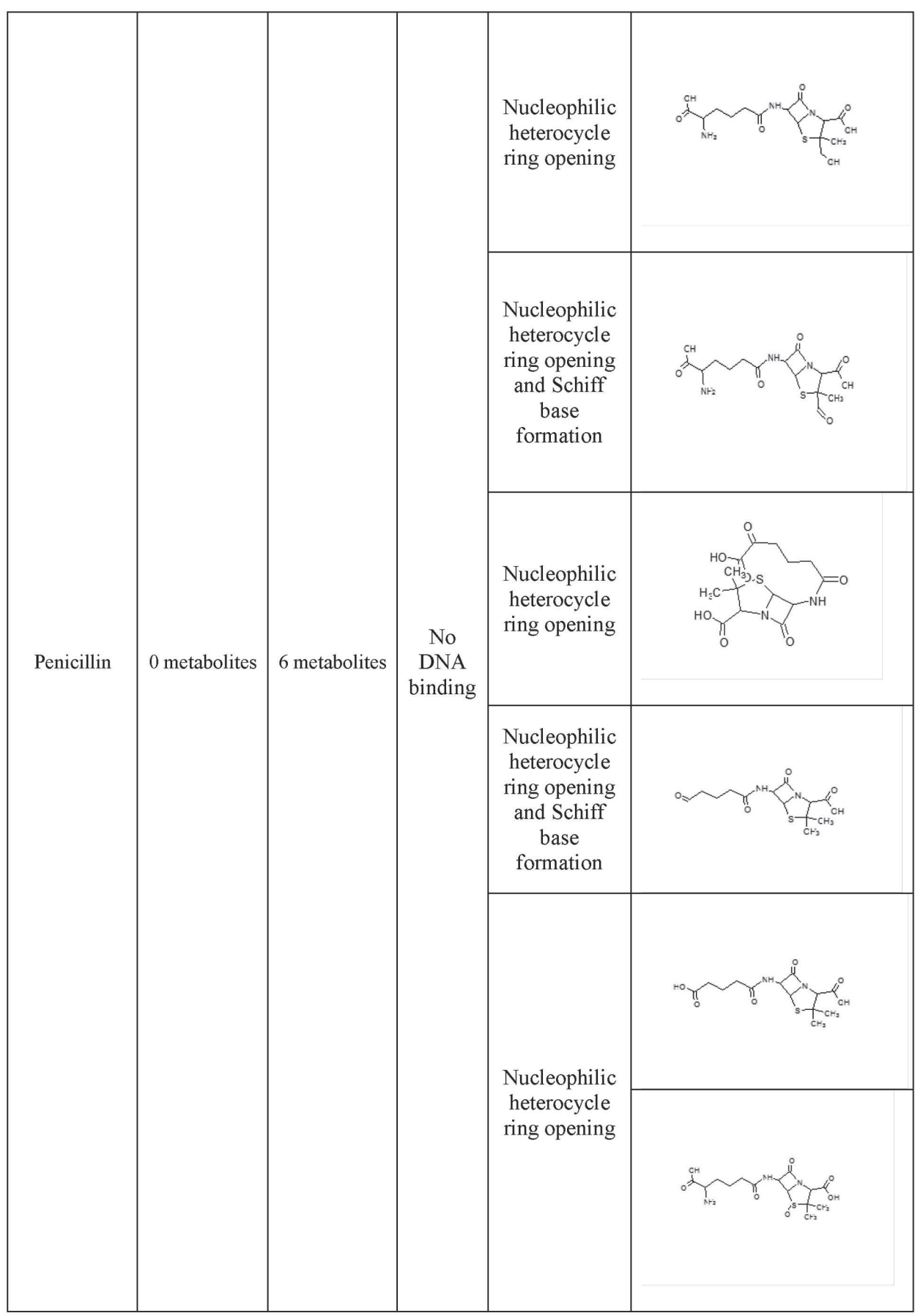


2. Bertolami, M. C. Mecanismos de hepatotoxicidade.- Arq. Bras. Cardiol., 85, 2005, Suppl. 5, 25-27.

3. Chang, C. Y., T. D. Schiano. Review article: drug hepatotoxicity.- Aliment. Pharmacol. Ther., 25, 2007, No 10, 1135-1151.

4. ChemIDPlus website.- http://chem.sis.nlm.nih.gov/ chemidplus

5. Galan, M. V., J. A. Potts, A. L. Silverman, S. C. Gordon. The burden of acute nonfulminant druginduced hepatitis in a United States tertiary referral center.- J. Clin. Gastroenterol., 39, 2005, No 1, 64-67.

6. Kaplowitz, N., T. Y. Aw, F. R. Simon, A. Stolz. Drug-induced hepatotoxicity.- Ann. Intern. Med., 104, 1986, No 6, 826-839.

7. Kaplowitz, N. Biochemical and cellular mechanisms of toxic liver injury.- Semin. Liver Dis., 22, 2002, No 2, 137-144.

8. Kaplowitz, N. Idiosyncratic drug hepatotoxicity.Nat. Rev. Drug Discov., 4, 2005, No 6, 489-499.

9. Kaplowitz, N. Rules and laws of drug hepatotoxicity.- Pharmacoepidemiol. Drug Saf., 15, 2006, No 4, 231-233.

10. Knowles, S. R., J. Uetrecht, N. H. Shear. Idiosyncratic drug reactions: the reactive metabolite syndromes.- Lancet, 356, 2000, No 9241, 1587-1591.

11. Larrey, D., S. Erlinger. Drug-induced cholestasis.Baillieres Clin. Gastroenterol., 2, 1988, No 2, 423-452.
12. Lee, W. M. Drug-induced hepatotoxicity.- New Engl. J. Med., 333, 1995, No 17, 1118-1127.

13. Mekenyan, O. G., S. D. Dimitrov, T. S. Pavlov, G. D. Veith. A systematic approach to simulating metabolism in computational toxicology. I. The TIMES heuristic modeling framework.- Curr. Pharm. Des., 10, 2004, No 11, 1273-1293.

14. Miller, J. A. The metabolism of xenobiotics to reactive electrophiles in chemical carcinogenesis and mutagenesis: a collaboration with Elizabeth Cavert Miller and our associates.- Drug Metab. Rev., 30, 1998, No 4, 645-674.

15. OECD (Q)SARs Application Toolbox.- http://www. oecd.org/document.en.html

16. Ostapowicz, G., R. J. Fontana, F. V. Schiødt, A. Larson, T. J. Davern, S. H. Han, et al.; U.S. Acute Liver Failure Study Group. Results of a prospective study of acute liver failure at 17 tertiary care centers in the United States.- Ann. Intern. Med., 137, 2002, No 12, 947-954.

17. Pearson, R. G., J. Songstad. Application of the principle of hard and soft acids and bases to organic chemistry.- J. Am. Chem. Soc., 89, 1967, No 8, 1827-1836.

18. SOM 208 Microbiology Syllabus.- http://www. ctcd.edu/science/tanderson/documents/ Micro_ Antibiotics.pdf 